This item was submitted to Loughborough's Research Repository by the author.

Items in Figshare are protected by copyright, with all rights reserved, unless otherwise indicated.

\title{
Loss aversion in an agent-based asset pricing model
}

\section{PLEASE CITE THE PUBLISHED VERSION}

https://doi.org/10.1080/14697688.2019.1655784

\section{PUBLISHER}

Taylor \& Francis

\section{VERSION}

AM (Accepted Manuscript)

\section{PUBLISHER STATEMENT}

This is an Accepted Manuscript of an article published by Taylor \& Francis in Quantitative Finance on 01 Nov 2019, available online: http://www.tandfonline.com/10.1080/14697688.2019.1655784.

\section{LICENCE}

CC BY-NC-ND 4.0

\section{REPOSITORY RECORD}

Pruna, Radu T., Maria Polukarov, and Nick Jennings. 2019. "Loss Aversion in an Agent-based Asset Pricing Model”. Loughborough University. https://hdl.handle.net/2134/16929844.v1. 


\title{
Loss Aversion in an Agent-Based Asset Pricing Model
}

\author{
Radu T. Pruna ${ }^{1}$, Maria Polukarov², Nicholas R. Jennings ${ }^{3}$ \\ ${ }^{1}$ School of Electronics $\&$ Computer Science, University of Southampton, UK \\ 2 Department of Informatics, King's College London, UK \\ 3 Departments of Computing and Electrical and Electronic Engineering, Imperial College London, UK
}

\begin{abstract}
A well-defined agent-based asset pricing model able to match the widely observed properties of financial time series is valuable for testing the implications of various biases associated with investors' behaviour. Extending one of the most successful models in capturing traders behaviour, we present a new behavioural agent-based asset pricing model. Specifically, we introduce a well-known behavioural bias in the model, loss aversion, and evaluate its implications. First, measuring how close the simulated time series are to its empirical counterparts, we show that the model with loss aversion better matches and explains the properties of real-world financial data, compared with the base model without the behavioural bias.

Secondly, we assess the impact of different levels of loss aversion not only on the agents' switching mechanisms, but also on the properties of the time series generated by the model. We demonstrate how for different levels of the loss aversion parameter, the biased agents tend to be driven out of the market at different points in time. Since even the simplest strategies have been shown to survive the competition in an agent-based setting, we can link our findings with the behavioural finance literature, which states that investors' systematic biases lead to unexpected market behaviour, instabilities and systematic errors.

Finally, we provide an in-depth analysis of the simulated time series and show the resulting dynamics replicate a rich set of the stylized facts including: absence of autocorrelation, heavy tails, volatility clustering and conditional heavy tails of returns, long memory of absolute returns, as well as volume-volatility relations, gain-loss asymmetry, power-law behaviour and long memory of volume.
\end{abstract}

Keywords: Agent-based modelling, asset pricing, loss aversion, behavioural bias, stylized facts

\section{Introduction}

A financial market is a broad term describing a marketplace where buyers and sellers participate in the trade of various financial assets at prices that reflect supply and demand. Since its introduction in the sixties by Samuelson (1965) and Fama (1970), from two rather different research perspectives, the Efficient Market Hypothesis (EMH) has become one of the most influential ideas in modern financial markets. There are weak and strong forms of the EMH, but when researchers refer to markets as being efficient, they usually mean that asset prices and returns are the outcome of a competitive market consisting of rational traders who try to maximise their returns. It postulates that prices reflect all available information, meaning that they should adjust instantly and correctly to incorporate new information.

In contrast, Keynes (1937) questioned the completely rational valuation of the assets, arguing that investors' sentiment and psychology, the so-called animal spirits, play a significant role in financial markets. The attacks on both theoretical and empirical sides of efficient markets ideas continued over the years, with most of the debates regarding market efficiency and rationality remaining unresolved (for a survey see, for example, Lo and MacKinlay (1988) and Lo (2007)). Full rationality requires all the agents to have knowledge about the beliefs of all the other participants in the market, thus making it impossible in a heterogeneous framework where traders have different expectations about future prices. On the other hand, heterogeneity obviously complicates the market models, making their analytical tractability almost 
impossible. A computational approach is therefore suited for investigating the heterogeneous world, offering a strong motivation for developing agent-based models of financial markets (Chen et al., 2012).

Generally speaking, agent-based models are systems in which a number of different agents interact with each other and their environment (Wooldridge and Jennings, 1995). Specifically, we can view financial markets as examples of evolutionary systems populated by agents with different trading strategies. Recently, we have witnessed a transition in economics and finance from the rational agent approach to agent-based markets populated by heterogeneous agents with bounded rationality (Hommes, 2006). In this context, the term bounded rationality suggests that individuals are not capable of the optimization levels required by full rationality. Instead, because of their limitations, market participants make choices that are satisfactory, but not necessarily optimal (Simon, 1955). In the last decades, an increasing number of heterogeneous agent models with bounded rationality have been introduced in the economics and finance literature (for recent surveys see Chiarella et al. (2008); Hommes (2006); LeBaron (2006)). This comes as an alternative to the rational expectations framework where all agents have fully, unbounded rationality.

Furthermore, models that rely on simple trading agents have proven themselves very efficient in generating important dynamics of real financial markets (Lux and Marchesi, 2000; Alfarano et al., 2005; Chiarella et al., 2006). One of the most influential model frameworks, the fundamentalists vs. chartists (to be defined), incorporates all the significant mechanisms of the markets, is capable of recreating their key properties and is simple enough for analysis and computation. In these models, fundamentalists act as a stabilizing force in the market and believe in the existence of a fundamental value. In contrast, chartists (also known as technical analysts), forecast the future prices entirely by modelling historical data. ${ }^{1}$ They do not take into consideration the market fundamentals and base their decisions on observed historical patterns in past prices.

However, researchers do not only try to illustrate the basic mechanisms of the models, but also quantitatively recreate the statistical properties of financial markets. To this end, independent studies have revealed a set of statistical properties common across different financial instruments, markets and time periods. These so-called stylized facts (Cont, 2001) target a wide range of aspects in the market, from price series and returns, to trading volume and volatility. Specifically, heterogeneous agent-based models (ABM) that use simple trading strategies have successfully generated a rich set of key properties that match the dynamics of asset prices in real financial markets (see Chen et al. (2012) for a review). They can provide useful insight into the behaviour of individual agents and also on the effects that emerge from their interaction, making the financial markets a very appealing application for agent-based modelling.

In recent years, a considerable focus has shifted from the econometric analysis of financial data towards developing models of human psychology as it relates to financial markets (Shiller, 2003). Behavioural finance follows the idea that investors behave according to their beliefs, which are updated based on new information. In contrast to other theoretical frameworks, behavioural finance studies the nature of financial judgements made by individuals and examines their consequences on the markets, while better understanding the updating processes of human beliefs. Since market participants only have limited information and restricted processing capabilities, they may have biases and deviate from rationality, which has, in turn, a large impact on price changes. These biases, also known as mental heuristics (Tversky and Kahneman, 1974), offer an explanation for such deviations from rationality (Kahneman, 2011).

In more detail, behavioural finance studies the most relevant psychological aspects of investors' decision making. There are a wide range of factors to be considered, from situational or personality-related, to entirely non-economic ones, associated to weather or other external events. Human decisions are the product of emotion, habit, reason and instinct and how these factors interact with each other depends on time, place and circumstances (Brennan and Lo, 2011). The main findings of behavioural finance are described in the work of Thaler (2005), Shefrin (2002) and De Bondt (2005), to name a few.

However, even though these biases have been clearly observed in real life investors' behaviour or identified in laboratory experiments, examining their implications on the overall market is still a challenging topic. Because of the high complexity of financial markets, it is almost impossible to assess the macro effects of a

\footnotetext{
${ }^{1}$ Surveys that brought to the attention of academics the use of technical analysis were conducted by Frankel and Froot (1990a,b), Taylor and Allen (1992) and more recently by Gehrig and Menkhoff (2004).
} 
particularly interesting micro dynamic. For this reason, it is hard to directly quantify the impact of various behavioural heuristics on the interaction between market participants or on the observed time series.

On the other hand, a well-defined agent-based model able to match the properties of financial markets is ideal for testing various behavioural and economical theories. We believe that the fields of agent-based modelling and behavioural finance complement each other and can be used together to provide a deeper understanding on the highly nonlinear economic systems. Not only can the agent-based framework serve as a useful theoretical tool for verifying the findings from behavioural finance, but it can also provide insights which are otherwise hard to observe in the real financial markets. Unfortunately, there are only a few models in the literature that combine the findings of the two fields. In this paper, we present one such behavioural agent-based model that incorporates an important bias in traders' behaviour.

In more detail, we build on the well-known structural stochastic volatility model of Franke and Westerhoff (2012), one of the most successful models in capturing the empirically observed traders' behaviour (Barde et al., 2015), to test the implications of loss aversion. This is one of the most researched behavioural heuristics observed in real life investors and it was shown to effectively explain a number of financial properties of stock returns (Barberis et al., 1999). Loss aversion offers an explanation to investors' unwillingness to realise and cut their loses. In other words, losses loom larger than gains (Tversky and Kahneman, 1991). In general, an individual is loss averse if he or she dislikes symmetric 50-50 bets and, furthermore, the preference increases with the size of the stakes. This concept applies when one is trying to avoid a loss even if it means accepting a higher risk.

We can relate the present contribution to the work of Chiarella and He $(2002,2003)$, who show how asset price dynamics are influenced by the relative risk attitudes of different types of investors. They depart from previous models that assume that all agents have the same risk attitude and consider a more heterogeneous world where agents are allowed to have different attitudes towards risk. Similar to their work, we relax the assumption in the FW model that all agents are loss neutral and include the empirically proven concept of loss aversion.

Our new setting is able to produce several interesting results that have not previously been seen in an agent-based model. Specifically, we focus on the overall implications of loss aversion and make the following contributions:

- Measuring how close a model is to its empirical counterparts, we demonstrate that introducing loss aversion leads to a new behavioural model that matches the properties of financial time series to a higher degree, compared with the model without loss aversion.

- We show that for certain degrees of loss aversion, the biased agents are driven out of the market. Since even zero-intelligence strategies have been shown to survive the competition in an agent-based setting (Ladley, 2012), we argue that it's the behavioural bias that leads to this misbehaving in the market.

- We test the effects of different levels of loss aversion on the time series generated by the model. We show that for various levels of loss aversion some of the biased agents disappear from the market and the returns series generated by the model deviate from their normal behaviour, having an increased peakiness and heaviness of the tails. We can relate these findings to the behavioural finance literature by showing that investors' biases lead to unexpected market behaviour, instabilities and systematic errors.

- We are able to recreate the well-known stylized facts of volatility clustering, heavy tails and absence of autocorrelation of returns. In addition, the conditional heavy tails in returns are matched for the first time by an ABM and, for the first time in a 2-type design model, we match the volume-volatility relations, gain-loss asymmetry, power-law behaviour and long memory of volume.

The remainder of the paper is organised as follows. We formally define the agent-based model in Section 2, followed by an estimation of its parameters in Section 3. In Section 4 we show that introducing loss aversion leads to a model that better explains the properties of financial data. Furthermore, in Section 5 we discuss the model's inner working and the impact of the loss aversion on the overall system. Next, in Section 6, we present our results. We give a thorough econometric analysis of the time series generated by 
the agent-based model, integrating a wide range of tests and evidence that demonstrate the presence of new stylized facts. Section 7 concludes.

\section{Model formulation}

In this section, we extend the fundamentalists vs. chartists asset pricing model of Franke and Westerhoff (2012) (FW) to capture loss aversion, which is one of the most researched behavioural biases of investors. In Section 2.1 we specify the model's price adjustments, as they were set in the original FW setting. Next, in Section 2.2 we define agents' interactions, building on the FW model to accommodate the introduction of loss aversion.

\subsection{Price adjustments}

The asset prices are determined by excess demand ${ }^{2}$, following some of the most prominent examples in the literature (Beja and Goldman, 1980; Chiarella, 1992). Here, by excess demand we mean the absolute positive or negative orders per trading period. The demand itself is kept as simple as possible, in the form of the demand per average trader. For fundamentalists, the demand is inversely related to the difference between the current price and the fundamental value. This is a natural choice since fundamentalists believe that the price will eventually mean revert to its perceived fundamental value. In particular, at time $t$ their core demand $D_{t}^{f}$ is proportional to the gap $p_{t}^{f}-p_{t}$, where $p_{t}$ is the log price of the asset at time $t$, while $p_{t}^{f}$ is the fundamental log value at time $t$. The fundamental value is considered to follow a geometric Brownian motion with drift $\mu_{p}$ and volatility $\sigma_{p}$, whose values will be formally estimated together with all the other model parameters. This modification was shown to overcome the stationary of price series generated by the FW model (Pruna et al., 2016). Similarly, the core demand of the chartists' group, $D_{t}^{c}$, is proportional to the price changes they have just observed, $\left(p_{t}-p_{t-1}\right)$, where $p_{t}$ and $p_{t-1}$ are the log prices at time $t$ and $t-1$, respectively.

The wide variety of within-group specifications are captured by noise terms added to each of the core demands. These terms encapsulate the within-group heterogeneity and scale with the current size of the group. Specifically, the noise is represented by two normally distributed random variables $\epsilon_{t}^{f}$ and $\epsilon_{t}^{c}$ for fundamentalists and chartists, respectively. In other words, one can think of the noise variables as a convenient way of capturing the heterogeneity of markets populated by hundreds or thousands of different agents. Each of the two noise terms are sampled at every iteration and added to the deterministic part of the demand, leading to the total demand per agent within the corresponding group.

Thus, combining the deterministic and stochastic elements, we get the net demand of each group for the asset in period $t$ as

$$
\begin{array}{lll}
D_{t}^{f}=\phi\left(p_{t}^{f}-p_{t}\right)+\epsilon_{t}^{f} & \epsilon_{t}^{f} \sim \mathcal{N}\left(0, \sigma_{f}^{2}\right) & \phi>0, \epsilon_{f}>0, \\
D_{t}^{c}=\chi\left(p_{t}-p_{t-1}\right)+\epsilon_{t}^{c} & \epsilon_{t}^{c} \sim \mathcal{N}\left(0, \sigma_{c}^{2}\right) & \chi \geq 0, \epsilon_{c} \geq 0,
\end{array}
$$

where $\phi$ and $\chi$ are constants denoting the aggressiveness of traders' demand and $\sigma_{t}^{f}$ and $\sigma_{t}^{c}$ are independent noise variances.

The agents are allowed to switch strategies at each iteration, so their market fractions fluctuate over time. Let $n_{t}^{f}$ and $n_{t}^{c}$ be fractions of fundamentalists and chartists in the market at time $t$, respectively.

The total demand $D_{t}$ is given by the equation:

$$
D_{t}=n_{t}^{f} D_{t}^{f}+n_{t}^{c} D_{t}^{c}
$$

Generally, $D_{t}$ will be in disequilibrium. That is, the total demand of the agents will not add up to zero and we will have an excess of either supply or demand. The actual market fractions will be determined by a logit model (to be defined), that takes into consideration various factors that might make an agent switch strategies (see Equation 9). Now, following some prominent examples in the literature (Farmer and Joshi,

\footnotetext{
${ }^{2}$ For an extensive empirical study on asset price changes caused by excess demand see Asparouhova et al. (2003).
} 
2002; Chiarella and He, 2003), a market maker is assumed to absorb the excess supply and provide any excess demand. The market maker mediates the transactions between investors and provides liquidity. He sets the price by supplying stock out of its inventory and raising the price if there is excess demand, while accumulating stock and lowering the price when there is excess supply. Specifically, the market maker reacts to the imbalance between demand and supply by proportionally adjusting the price with a constant factor $\mu>0$.

Accordingly, the equation determining the price for the next period $t+1$ results from equations (1)-(3) as

$$
\begin{gathered}
p_{t+1}=p_{t}+\mu\left[n_{t}^{f} \phi\left(p_{t}^{f}-p_{t}\right)+n_{t}^{c} \chi\left(p_{t}-p_{t-1}\right)+\epsilon_{t}\right], \\
\epsilon_{t} \sim \mathcal{N}\left(0, \sigma_{t}^{2}\right), \quad \sigma_{t}^{2}=\left(n_{t}^{f} \sigma_{f}\right)^{2}+\left(n_{t}^{c} \sigma_{c}\right)^{2} .
\end{gathered}
$$

Equation (5) is derived as the sum of the two normal distributions from Equations (1) and (2), multiplied by the market fractions $n_{t}^{f}, n_{t}^{c}$. The result is a new normal distribution with mean zero and variance equal to the sum of the two single variances. The combined variance $\sigma_{t}$ depends on the variations of the market fractions of the fundamentalists and chartists. This random time-varying variance is a key feature of the model, being termed the structural stochastic volatility of returns (defined as the log differences in prices) in Franke and Westerhoff (2011).

\subsection{Evolution of the market shares}

To complete the model, it is necessary to set up the dynamics of the market fractions $n_{t}^{f}$ and $n_{t}^{c}$. They are predetermined within each period and change only from one period to the next one. In this section, we modify the setting presented in the FW model to accommodate for our newly introduced behavioural factors. Following the discrete choice approach (DCA) introduced by Brock and Hommes (1997), the two market shares $n_{t+1}^{s}(s=f, c)$ can be determined using the multinomial logit model. In the basic setting, some payoff indices $u_{t}^{c}$ and $u_{t}^{f}$ are considered, usually derived from past gains of the two groups. The market fractions can be expressed as $n_{t+1}^{s}=\exp \left(\beta u_{t}^{s}\right) /\left[\exp \left(\beta u_{t}^{f}\right)+\exp \left(\beta u_{t}^{c}\right)\right]$, where $\beta$ is the intensity of choice. Dividing both numerator and denominator by $\exp \left(\beta u_{t}^{f}\right)$, the market fraction of fundamentalists is given by $n_{t+1}^{f}=1 /\left\{1+\exp \left[-\beta\left(u_{t}^{f}-u_{t}^{c}\right)\right]\right\}$.

Note that the difference in any utility variables, $\left(u_{t}^{f}-u_{t}^{c}\right)$, can be viewed as a measure of relative attractiveness of fundamentalist trading. Hence, we may change the notation, making use of the attractiveness level, $a_{t}$ (Franke and Westerhoff, 2012), defined as the difference $\left(u_{t}^{f}-u_{t}^{c}\right)$. The discrete choice approach is then given by

$$
n_{t+1}^{f}=\frac{1}{1+\exp \left(-\beta a_{t}\right)}, \quad n_{t+1}^{c}=1-n_{t+1}^{f} .
$$

We normalize all the demand terms in the price rule (Equation (4)) by using a market impact $\mu=0.01$. Moreover, we fix the intensity of choice $\beta=1$, which is just a matter of scaling the attractiveness level of fundamentalism, $a_{t}$. These values are suitable for modelling a daily time series.

Note that the market fractions are directly influenced by the attractiveness level. An increase in the index $a_{t}$ leads to an increase in the market share of the fundamentalists. For this reason, it is extremely important to define the exact mechanism of the attractiveness level and all of its components.

The concept of loss aversion is linked to a level of wealth, from which we can derive the domains of gains and losses (Tversky and Kahneman, 1992). In this context, the first principle that influences the agents' choice of strategies is based on differential wealth. In detail, with respect to strategy $s=f, c$, let $g_{t}^{s}$ be the short term capital gains that an average agent in this group could realise at time $t$. This is obtained from the demand formulated at time $t-2$ and executed at the price $p_{t-1}$ of the next day. In general, a utility $u_{t}^{s}$ obtained from these gains is defined as $u_{t}^{s}=g_{t}^{s}+\eta u_{t-1}^{s}$, where $\eta$ is a memory coefficient between 0 and 1 . Following the agents' wealth definition of Franke and Westerhoff (2012), we consider a weighted average of $g_{t}^{s}$ and $u_{t-1}^{s}$ such that

$$
\begin{array}{r}
g_{t}^{s}=\left[\exp \left(p_{t}\right)-\exp \left(p_{t-1}\right)\right] D_{t-2}^{s}, \\
w_{t}^{s}=\eta w_{t-1}^{s}+(1-\eta) g_{t}^{s} .
\end{array}
$$


Note that we replaced the utility symbol $u$ with $w$ to accommodate our interpretation. Furthermore, $w_{t}^{s}$ represents the accumulated profits, discounted by the coefficient $\eta<1$, that would have been earned by an agent who had consistently followed strategy $s$ on a daily basis. In other words, the accumulated wealth attributed to a particular strategy is similar to a utility of performance and will be used by agents only when considering their switching strategy.

According to prospect theory, loss aversion influences investors' overall risk attitude, making them riskaverse in the domain of gains but risk-seeking when the changes in wealth are perceived as losses (Tversky and Kahneman, 1992). In more detail, an individual is loss averse if there is an asymmetry in how he or she perceives gains and losses of the same magnitude. Specifically, losses loom larger than gains - that is, people weigh losses more than gains of similar magnitude. Our implementation of loss aversion is slightly different from prospect theory in the sense that agents are considering an asymmetry in wealth, instead of expected terms. Here, we are not arguing that our model of loss aversion is the only, or even the best, way to implement loss aversion. Rather, we introduce loss aversion in a way that can be supported by the overall model's definition, where wealth is similar to a utility of performance. Specifically, the agents choose their strategies (chartism vs. fundamentalism) based on how well they have worked over time. In this context, loss aversion is implemented in a way such that investors are affected by losses more than gains of the same magnitude, which in turn influences their switching behaviour. That is, we modify the agents' interactions by introducing an asymmetric dependency on positive and negative payoffs. Therefore, loss averse agents consider an asymmetric wealth function such as

$$
v_{t}^{s}= \begin{cases}w_{t}^{s} & \text { if } w_{t}^{s} \geq 0 \\ \lambda w_{t}^{s} & \text { if } w_{t}^{s}<0\end{cases}
$$

where $\lambda$ is the loss aversion coefficient, $s=f, c$. Note that the asymmetric wealth, $v_{t}^{s}$, is only relevant for the attractiveness level, $a_{t}$, defined by Equation 9, when the performance of chartism is compared with fundamentalism. Now, in their seminal paper, Tversky and Kahneman (1992) argue that people weigh losses twice as much as gains of a similar magnitude, fixing the loss aversion coefficient at $\lambda=2.25$. In Section 3 we will see how different values of this influence the overall system and interaction between agents. Finally, we introduce the first term in the attractiveness level $a^{t}$ as being proportional to the difference in accumulated wealth, $\left(v_{t}^{f}-v_{t}^{c}\right)$.

The second principle considered is based on one of the most empirically observed social factors in behavioural literature, known as herding (for a review see Bikhchandani and Sharma (2000)). This is the investors' tendency to follow the strategies of other traders. It is one of the essential irreducible elements that an agent-based model of financial markets should posses (Alfi et al., 2009). The basic idea of herding is that joining a group becomes more attractive the more adherents it already has. In our model, this principle will be represented by a term proportional to the most recent difference between market fractions, $\left(n_{t}^{f}-n_{t}^{c}\right)$.

The last term of the attractiveness level is based on a predisposition towards one of the two strategies. This is simply expressed by a constant factor, $\alpha_{0}$, which is positive (negative) if there is a priori preference toward fundamentalism (chartism). These three elements combined form the attractiveness level, formally defined as

$$
a_{t}=\alpha_{0}+\alpha_{n}\left(n_{t}^{f}-n_{t}^{c}\right)+\alpha_{w}\left(v_{t}^{f}-v_{t}^{c}\right)
$$

where $\alpha_{0}$ is the predisposition parameter, $\alpha_{n}>0$ captures the herding parameter and $\alpha_{w}>0$ measures the influence of accumulated wealth.

\section{Estimation of the model's parameters}

In this section we discuss the formal numerical estimation of the model's parameters, using one of the most prominent methods from the literature, the method of simulated moments (MSM) (see e.g. (Gilli and Winker, 2003; Chen and Lux, 2018). An agent-based model is not supposed to mimic the exact economy or financial markets, but rather it should explain some of the most important stylized facts of financial markets, making this estimation method suitable for our purposes. This technique is based on an objective 
Table 1: Model parameters

\begin{tabular}{llllllllll}
\hline$\phi$ & $\chi$ & $\sigma_{f}$ & $\sigma_{c}$ & $\alpha_{0}$ & $\alpha_{n}$ & $\alpha_{w}$ & $\mu_{p}$ & $\sigma_{p}$ & $\eta$ \\
\hline 1.00 & 0.912 & 0.733 & 1.760 & 2.127 & 1.277 & 2679 & 0.014 & 0.17 & 0.987 \\
\hline
\end{tabular}

function that is optimised across the set of model's parameters $\left(\phi, \chi, \sigma_{f}, \sigma_{c}, \alpha_{0}, \alpha_{n}, \alpha_{w}\right)$. The method refers to a set of statistics, also known as moments, arising from the simulations. The basic idea is that these moments should be close to their empirical counterparts, with the distance between them being captured by the objective function.

In this paper, we calibrate our behavioural model using the same dataset of $\mathrm{T}=6866$ daily observations of the Standard and Poor (S\&P 500) stock market index from January 1980 to mid-March 2007, as per the FW model. The estimation was conducted such that the four most discussed statistical properties of empirical financial data are matched. These include absence of autocorrelation in raw returns, heavy tails, volatility clustering and long memory.

In order to conduct the quantitative analysis, the four stylized facts were measured using a number of summary statistics, or moments. The first moment considered is the first-order autocorrelation coefficient of raw returns. It needs to be close to zero, so that it agrees with the empirical findings on this matter (Bouchaud and Potters, 2003; Cont et al., 2014). This will limit the chartists' price extrapolations of the most recent price changes. Furthermore, if the model generates coefficients that are insignificant, all the autocorrelations at longer time lags will vanish too.

The remaining three moments are dealing with the volatility of returns. First of all, the model should suitably scale the overall volatility, thus limiting the general noise brought by the two variances $\sigma_{f}$ and $\sigma_{c}$. The mean value of the absolute returns is considered. Next, the heavy tail is measured by the Hill tail index of the absolute returns, at the upper $5 \%$ level. The long memory effects are captured by the autocorrelation function $(\mathrm{ACF})$ of the absolute returns up to a lag of 100 days. In particular, the autocorrelation decays as we increase the lag, without becoming insignificant. The entire profile has to be matched and is sufficiently well represented by six different lag coefficients $(\tau=1,5,10,25,50,100)$ (Franke and Westerhoff, 2012).

Thus, the model is evaluated on the basis of nine moments (the Hill estimator, volatility, first order autocorrelation of the raw returns and the autocorrelation at lags $\tau=1,5,10,25,50,100$ for the absolute returns), summarized in a (column) vector $m=\left(m_{1}, \ldots, m_{9}\right)^{\prime}$ (the prime denotes transposition). According to the MSM, they have to come as close as possible to their equivalent empirical moments, $m^{e m p}$, calculated on the daily S\&P 500 stock market index.

The distance between the two vectors $m$ and $m^{e m p}$ is defined as a quadratic function given by

$$
J=J\left(m, m^{e m p} ; W\right)=\left(m-m^{e m p}\right)^{\prime} W\left(m-m^{e m p}\right) .
$$

The weight matrix $W$ accounts for the moments' sampling variability and its determination is crucial for the model's parameters. The idea is that the higher the sampling variability of a given moment $i$, the larger the differences between $m_{i}$ and $m_{i}^{e m p}$ that can still be considered insignificant. Moreover, the matrix $W$ should support possible correlations between single moments. One obvious choice for $W$ is the inverse of an estimated variance-covariance matrix $\hat{\Sigma}$ of the moments (Franke, 2009),

$$
W=\hat{\Sigma}^{-1} .
$$

The covariances in $\hat{\Sigma}$ are estimated by a bootstrap procedure used to construct additional samples from the empirical observations. Here, we depart from the block bootstrap usually used in the literature (Winker et al., 2007) and use a new bootstrap method which was shown to overcome the joint-point problem (for more details see Franke and Westerhoff (2016)). Specifically, we sample single days and, associated with each of them, the history of the past few lags required to calculate the lagged autocorrelations. For a good representation, the bootstrap method is repeated 5000 times, obtaining a distribution for each of the moments.

Going back to the estimation problem, we are interested in the set of parameters that minimise the distance function $J$ from Equation (10). Repeated simulations are carried out, in search of the set of 
parameters that minimise the associated loss. To this end, let $\theta$ be the vector of parameters and $m=m(\theta ; S)$ denote the moments to which a vector $\theta$ gives rise. Let $m^{a}(\theta, S)$ denote the moment vector obtained by simulating the model with a parameter vector $\theta$ over $S$ periods on the basis of a random seed $a=1,2 \ldots$ The parameter estimates on a random seed $a$, denoted $\hat{\theta}^{a}$, are the solution of the following minimisation problem,

$$
\hat{\theta}^{a}=\underset{\theta}{\operatorname{argmin}} J\left[m^{a}(\theta ; S), m_{T}^{e m p}\right],
$$

where $m_{T}^{e m p}$ is the moment vector for the empirical S\&P 500 data.

Although one may think that a simulation over $S=68660$ days provides a large sample to base the moments on, the variability arising from such different samples is still considerable. Hence, we run a Monte Carlo method by carrying out a great number of estimations $(a=1000)$ and choosing the one with the median loss. The parameter set $\hat{\theta}$ giving this associated loss will be our representative estimation. Specifically, using Equation (12) we have $\hat{\theta}=\hat{\theta}^{\tilde{a}}$, where $\tilde{a}$ is such that $\hat{J}^{\tilde{a}}$ is the median of $\left\{\hat{J}^{a}\right\}_{a=1}^{1000}$ and $\hat{J}^{a}=J\left[m^{a}\left(\hat{\theta}^{a} ; S\right), m_{T}^{e m p}\right]$, with $a=1, \ldots, 1000$. The minimised loss,

$$
\hat{J}=J\left[m^{\tilde{a}}(\hat{\theta} ; S), m_{T}^{e m p}\right]=7.178
$$

corresponds to the final parameter vector $\hat{\theta}$ resulting from the estimation (see Table 1 ). Note that the drift and volatility of fundamental price refer to a yearly time scale.

\section{Model evaluation}

In this section we are interested in assessing the quality of the moment matching achieved by our model with loss aversion compared to the some model without the behavioural bias. After a straightforward estimation has given us the numerical values for the model's parameters, we investigate if it would be rejected by real data. This way we demonstrate one of the central objectives of an agent-based financial model, namely how good it is at matching and explaining the properties of real world data.

In more detail, we use two different ways of measuring the model's goodness-to-fit. In Section 4.1 a p-value is derived, showing that the model with loss aversion generates data that has a higher chance of being mistaken to a real-world time series than the same model without loss aversion. Secondly, in Section 4.2 we take into account how often the simulated returns yield properties that are contained within their empirical confidence intervals. Here, using a second quantitative measure we demonstrate that introducing loss aversion in agents' behaviour leads to a model that better matches the statistics of empirical data.

\subsection{Moment-specific p-value}

We start with the bootstrapped moments of empirical returns, previously used to derive an estimated variance-covariance matrix of the moments. The large sample of $B=5000$ vectors obtained $\left(m^{1}, m^{2}, \ldots, m^{B}\right)$ can now be used to apply the loss function to them, thus obtaining an entire frequency distribution for $J$. From this, we can get a critical value of $J$ and evaluate our model numerical simulations against it. The idea is that such a bootstrapped distribution proxies the one of a hypothetical real-world data generation process. Therefore, if a model-generated return series and its moments have a value of $J$ within the range of the bootstrapped $J$, we would not be able to differentiate it from a real-world time series. Accordingly, accounting for the rare events with a $5 \%$ significance level, a simulated return series will be considered inconsistent with the real-world data if its $J$-value exceeds the $95 \%$ quantile of the bootstrapped $J$.

Moreover, we cannot consider the minimised $J$ functions from Equation 12 since they were obtained over a simulation horizon $S$ that is 10 times longer than the bootstrapped returns. For a more meaningful comparison to their values, the $J$-values should come from a model simulation of same length $T=6866$. To this end, we perform a Monte Carlo experiment and repeat the simulations many times, giving rise to a distribution of model-generated $J$-vales that can be compared with the bootstrapped ones. 
Table 2: Empirical moments and their 95\% confidence intervals

\begin{tabular}{llllllllll}
\hline & $v$ mean & $r$ AC-1 & $v$ AC-1 & $v$ AC-5 & $v$ AC-10 & $v$ AC-25 & $v$ AC-50 & $v$ AC-100 & Hill \\
\hline Measured & 0.713 & 0.019 & 0.184 & 0.215 & 0.156 & 0.126 & 0.109 & 0.070 & 3.299 \\
\hline Lower bound & 0.648 & -0.003 & 0.093 & 0.150 & 0.103 & 0.072 & 0.030 & 0.004 & 3.054 \\
\hline Upper bound & 0.788 & 0.044 & 0.241 & 0.239 & 0.181 & 0.148 & 0.107 & 0.068 & 3.976
\end{tabular}

In more detail, let $m^{b}=m^{b}\left(r_{t}^{e m p}\right)_{t=1}^{T}$ be the bootstrapped moments given by the empirical returns. Furthermore, let $\hat{\theta}$ be the estimated vector of a model's parameters, giving rise to Monte Carlo moment simulations $m=m^{c}(\theta, T), \mathrm{c}=1, \ldots, 5000$. The two frequency distributions of $J$-values are given by

$$
\begin{aligned}
\text { Bootstrap : } & J\left[m^{b}\left(\left(r_{t}^{e m p}\right)_{t=1}^{T}\right)\right]_{b=1}^{5000} \\
\text { MonteCarlo: } & J\left[m^{c}(\hat{\theta}, T)\right]_{c=1}^{5000 .}
\end{aligned}
$$

We can now compare the performance of our model with loss aversion with the FW model without loss aversion. After obtaining the critical value $J_{0.95}$ of the bootstrapped loss function, we calculate its analogous quantile in the two simulated distributions. While for the $\mathrm{FW}$ model without loss aversion $J_{0.95}$ corresponds to its $31.06 \%$ quantile, introducing loss aversion with a coefficient $\lambda=2.25$ in agents' behaviour leads to a higher $32.76 \%$ quantile (see Table 3 ). For a clear summary of the general moment matching we can say that the model has a p-value of $32.76 \%$, with respect to parameters $\hat{\theta}$ from Table 1 , the data of the $\mathrm{S} \% \mathrm{P}$ 500 index, and the specific nine moments that we have chosen. In other words, in almost one third of the simulations it is not possible to reject our model. Moreover, the introduction of loss aversion leads to a higher p-value.

\subsection{Moment coverage ratios}

The previous section the model evaluation was based on the values of the objective function $J$. Now, we can use a more direct way to assess the degree of moment matching of a model, which also accounts for single moments, first described by Franke and Westerhoff (2012). In order to do this, we derive a confidence interval for the empirical moments from the bootstrapped ones $\left(m^{1}, \ldots, m^{B}\right)$, presented in Table 2. Intuitively, a simple qualitative assessment of a given return series is that it cannot be rejected as being incompatible with the data if all of its moments are contained in the confidence intervals.

However, since the sample variability of a single simulation is not sufficient to evaluate the model as a whole, we employ another Monte Carlo estimation. This leads to a so-called coverage ratio, defined as the percentage of Monte Carlo runs for which a single moment, or all moments jointly, are contained in their confidence intervals. As before, we are mainly interested in assessing the implications of loss aversion and we compute the moment coverage ratios for the model with loss aversion and the one without it. The central joint coverage ratios and individual ones are presented in Table 3.

In order to put this in a more quantitative perspective, consider the event sampling moments from a real world data generating process that fall into their confidence intervals. If we account for the fact that there exists certain dependence between them and only some are independent (say $r$ AC-1, Hill, $v$ Mean, $v$ AC-1 and $v \mathrm{AC}-100)$ then a true data generating process would have a joint MCR of $0.95^{5}=77.4 \%$. Using this as a conservative benchmark against our Monte Carlo ratio, the model without loss aversion corresponds to an effective MCR of 27.4/77.4 =35.40\%, and the model with loss aversion to an effective MCR of $37.05 \%$.

We should note that all these statements are based on asymptotic theory. It is unclear if the confidence intervals we derived are a good representation of the ones appropriate for small samples of a true data generating process. Under this constraint, we have used the bootstrapped empirical returns to get more information about the small sample properties. Once more, taking the 5000 bootstrap samples, we compute the joint MCR for them and use this as a benchmark against which we can measure our models' ratios (in percentage points). The result is a ratio of $51.80 \%$, which is considerably lower than the hypothetical reference of $77.4 \%$. On the other hand, the $51.80 \%$ is higher than the MCRs of the models, as expected. Now, the most obvious way to relate our models' MCR to that of the bootstrap is to express them as a 


\begin{tabular}{lll}
\multicolumn{3}{c}{ Table 3: Model evaluation } \\
\hline & Model without LA & Model with LA \\
\hline p-value & $\mathbf{3 1 . 0 6}$ & $\mathbf{3 2 . 7 6}$ \\
joint MCR & 27.40 & 28.68 \\
\% of joint MCR $\left\{r_{t}^{\text {emp }}\right\}$ & $\mathbf{5 2 . 8 9}$ & $\mathbf{5 5 . 3 7}$ \\
$v$ mean & 83.10 & 82.64 \\
$r$ AC-1 & 70.34 & 70.34 \\
$v$ AC-1 & 99.30 & 99.36 \\
$v$ AC-5 & 66.76 & 68.74 \\
$v$ AC-10 & 85.78 & 86.12 \\
$v$ AC-25 & 81.76 & 82.72 \\
$v$ AC-50 & 79.3 & 80.78 \\
$v$ AC-100 & 78.42 & 78.94 \\
Hill & 95.8 & 95.48
\end{tabular}

fraction of it. This is done in the third row of Table 3. Referring to these statistics, we see that the model with loss aversion reaches a level of $55.37 \%$, higher than the $52.89 \%$ of the same model without loss aversion.

Nonetheless, this measure is probably an overestimation of a more appropriate relative coverage statistic and should not be taken too literally (Franke and Westerhoff, 2012). In any case, irrespective of the measure we refer to, the degree of the model's ability to match the statistics of real data is remarkable, with a plus for the one with loss aversion demonstrated by both the p-value and the coverage ratio (Table 3).

\section{Behavioural implications}

We now turn to explore the qualitative measures that demonstrate the existence of the most important stylized facts of financial markets. An in-depth analysis of the price, returns, volatility and volume series generated by the behavioural model will be performed in Section 6. The numerical parameters used for all the simulations are given in Table 1. Moreover, the loss aversion parameter is fixed at $\lambda=2.25$, as reported by Tversky and Kahneman (1992).

In Figure 1 we present a simple run of the model over 6867 days, covering the same time span as the empirical data used in estimating the model's parameters. A quick visual inspection of the simulated price series and the majority index, defined as the difference in market fractions $n_{f}-n_{c}$, plotted in Figures 1 (a) and (b), respectively, reveals a high market fraction of chartism when the price $\left(p_{t}\right)$ deviates from its fundamental value $\left(p_{t}^{f}\right)$. On the other hand, the fundamentalists increase in number when the price gets closer to the fundamental value. This behaviour is obvious from the model definition. The wealth element in the attractiveness level equation makes fundamentalism more appealing when the price moves towards its fundamental value, making it a profitable strategy. An increasing number of fundamentalists begin to dominate the market and their actions start the mean reverting process of the price towards the fundamental value. As the current price gets too close to the fundamental value, fundamentalism is no longer attractive or profitable, making the agents switch to chartism. The herding element takes over, leading to a majority fraction of chartism, whose actions will move the price away from the fundamental value.

In addition, Figure 1 (c) plots the accumulated wealth, $w_{t}$, of the two classes of traders. We can observe that when the actual price comes closer to the fundamental value the fundamentalists are more profitable, while the chartists make bigger profits when prices move away from the perceived fundamental value. Finally, the fourth panel in Figure 1 demonstrates the implications of these irregular switches in the agents' strategies on returns. Note that the volatility or swings in chartists' wealth is expected to be higher than for fundamentalists, since they have a greater variability in demand. Similarly, by comparing Figures 1 (b) and (d) we observe that the level of returns during a chartism domination exceeds its level in a fundamentalism regime. Therefore, it appears that normal sequences of returns are interrupted by outbursts of increased volatility, when the majority of agents are chartists. 


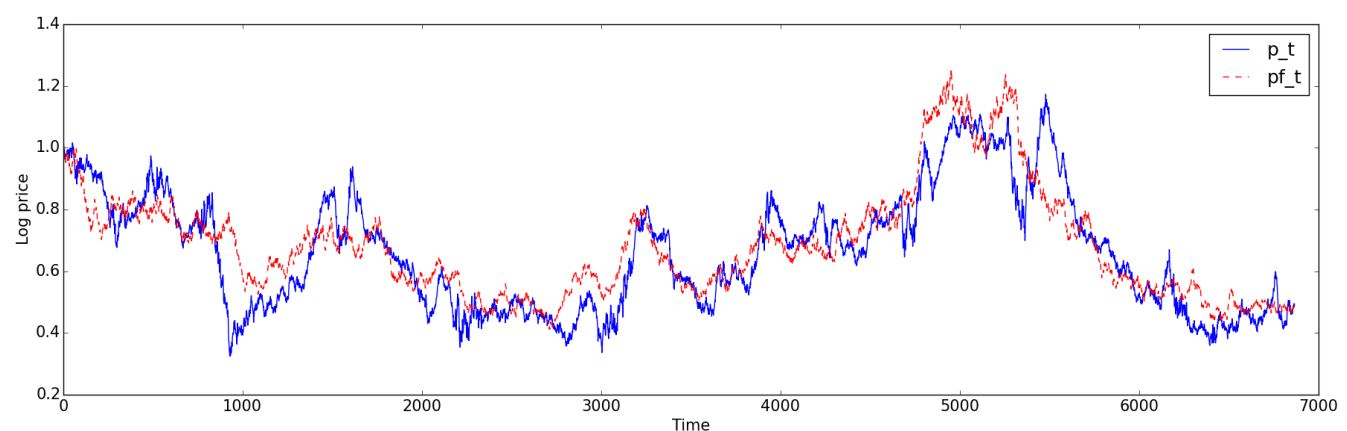

(a) Log prices

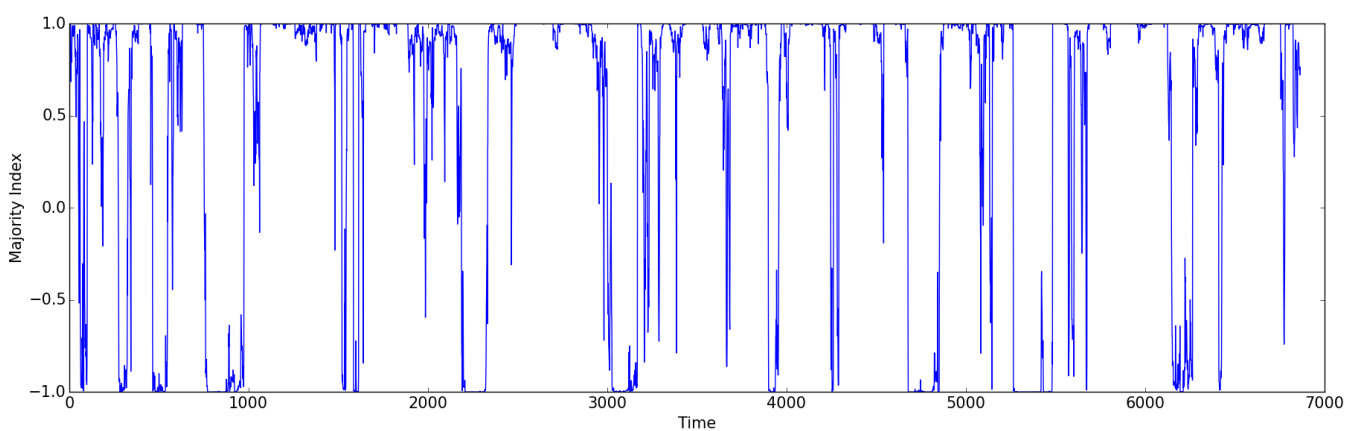

(b) Majority index

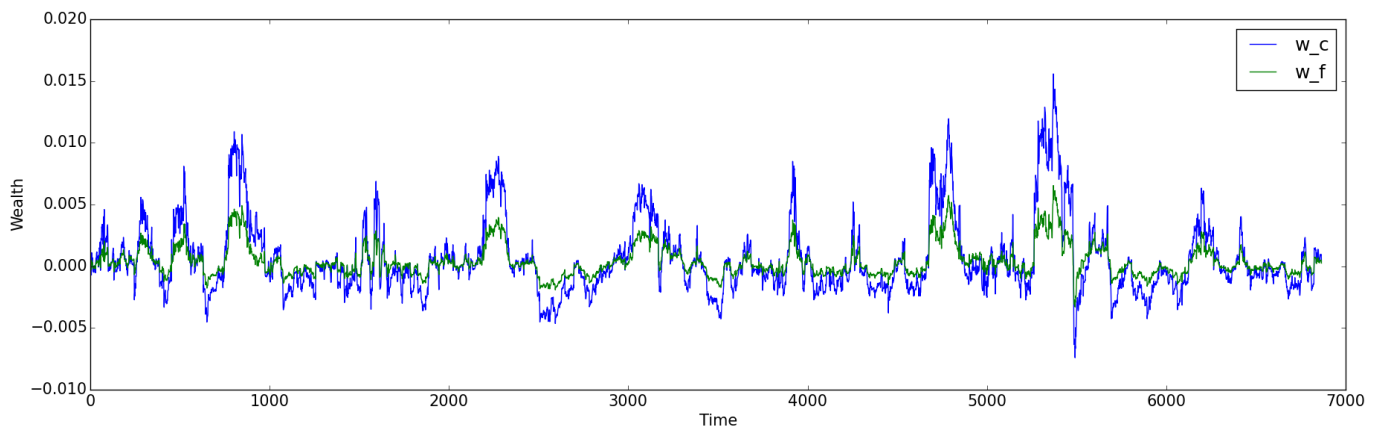

(c) Wealth

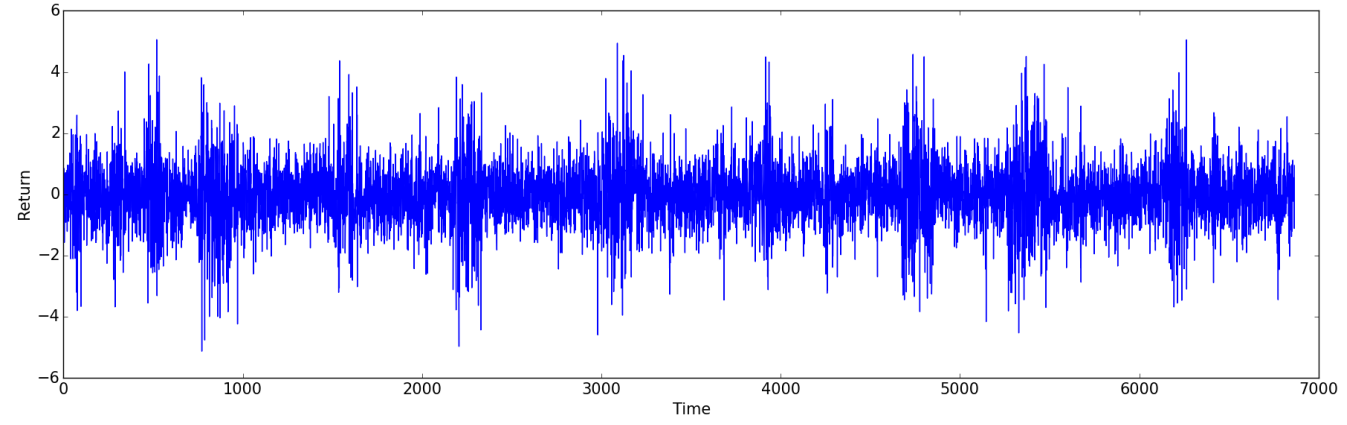

(d) Returns

Figure 1: Simulated price series, fundamental price series, majority index, wealth and returns over 6867 time periods. 


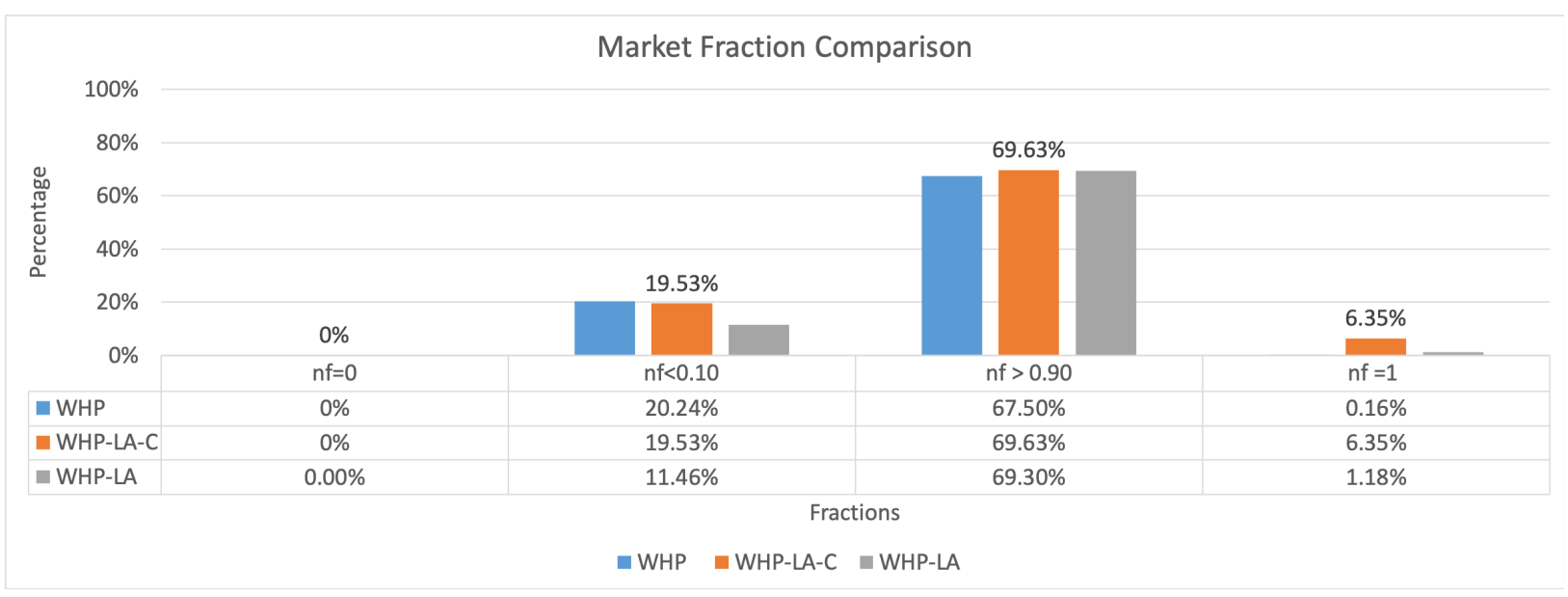

Figure 2: Fundamentalists' market fraction comparison via Monte-Carlo simulations for WHP, WHP-LA and WHP-LA-C models.

Clearly, our agent-based model is able to produce the real life dynamics of both price and return series. By inspecting Figure 1, we can observe the apparent randomness of the data, similar to what we witness in the real financial markets. However, our aim here is not just replicate the real life dynamics, but use such a model to test the implications of loss aversion. In the next section we will show how this behavioural bias influences the interactions between agents and the results reproduced by our model.

\subsection{Impact of loss aversion}

In order to assess the impact of loss aversion to the interaction between agents, we perform a more indepth analysis of the market fractions. For an accurate comparison, we consider 3 models that differ just in this respect. Namely, we compare our previously described behavioural model with loss aversion with the same model but without this bias in agents' behaviour. In particular, we look at the market fractions for three models, all having the switching mechanism dependent on wealth $(\mathrm{W})$, herding $(\mathrm{H})$ and a priori predisposition towards one of the two strategies $(\mathrm{P})$. The first model to consider has the behavioural presence of loss aversion only for chartists (WHP-LA-C), the second model considers both fundamentalists and chartists loss aversion (WHP-LA) and the third one doesn't have the loss aversion bias at all (WHP).

For a more robust comparison, we perform a Monte Carlo analysis considering 1000 simulation runs for WHP, WHP-LA and WHP-LA-C models and look at the fundamentalists' market fraction $\left(n_{f}\right)$ results, reported in Figure 2. As we have mentioned earlier, the market fractions stay at the ends of the interval, with the market being controlled by either chartists or fundamentalists, at different points in time. For all three models, we observe the market is heavily dominated by the fundamentalists $\left(n_{f}\right.$ median $\left.=0.99\right)$ with sudden disruptions of chartism, $n_{f}<0.1$ in more than $10 \%$ of the simulations.

At first glance, the market fractions appear to be similar in our three models, WHP, WHP-LA and WHP-LA-C. However, a closer look at the ends of the interval reveals major differences between the three settings. While fundamentalists never disappear from the market $\left(n_{f}>0\right)$, we notice that for WHP-LA-C model, the chartists can be driven out of the market ${ }^{3}$. Indeed, the fundamentalists tend to occupy the entire market $\left(n_{f} \rightarrow 1\right)$ in quite a few cases. In addition, over a median simulation of $T=6867$ time

\footnotetext{
${ }^{3}$ Note that for any positive finite level of the intensity of choice parameter $\beta$, a non-zero fraction of both classes of agents will always be in the market. However, in a setting where the market moves against the loss averse chartists for an extended period of time, they will completely overestimate their wealth and its effect on them. Therefore, their perceived level of wealth will be much lower than the actual one (see Equation 8). In turn, this leads to big differences in opinion that determine the attractiveness level in Equation 9 and the market fractions in Equation 6. The exponential function from Equation 6 will then tend to zero and the fundamentalists' fraction $n_{f}$ will tend to 1 . In such cases, we say that the chartists are driven out of the market.
} 


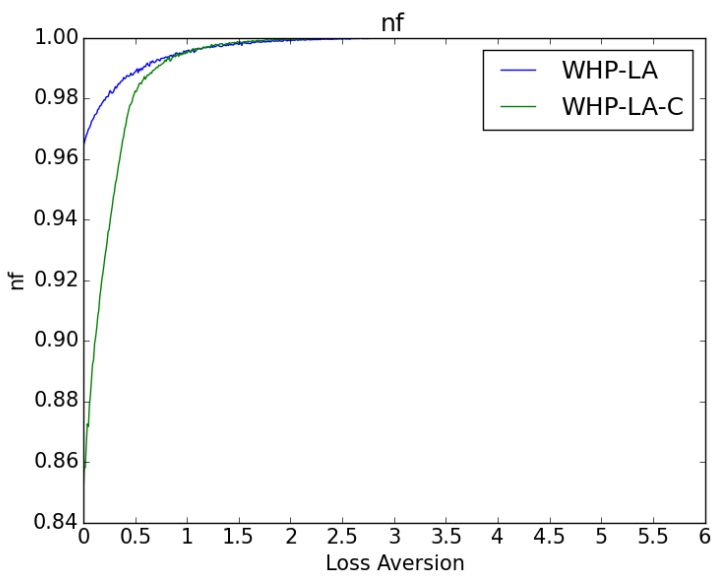

(a) Fundamentalists market fractions

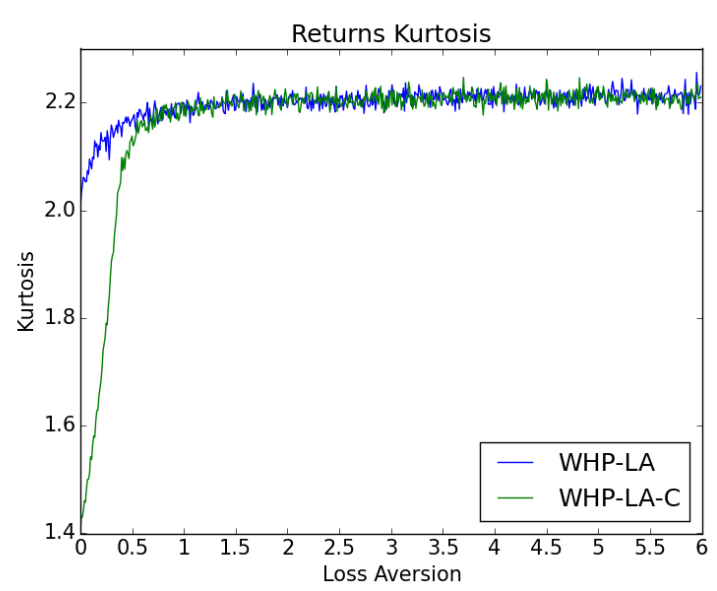

(c) Kurtosis

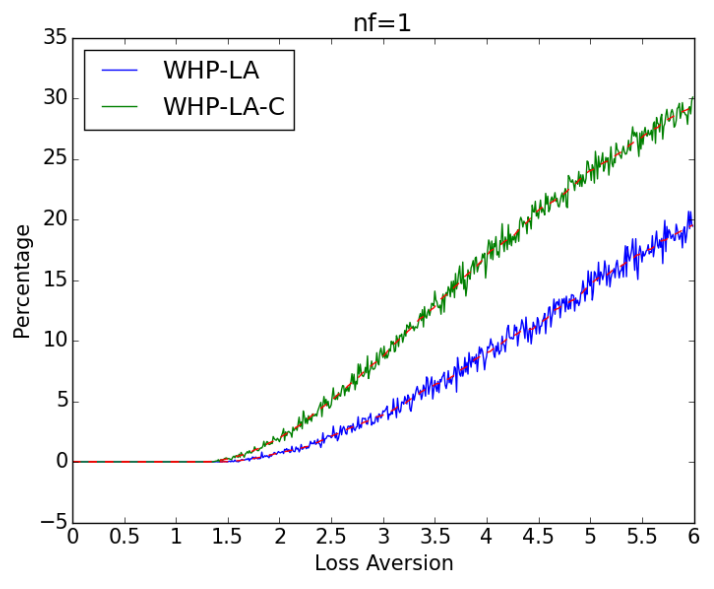

(b) Fundamentalists domination

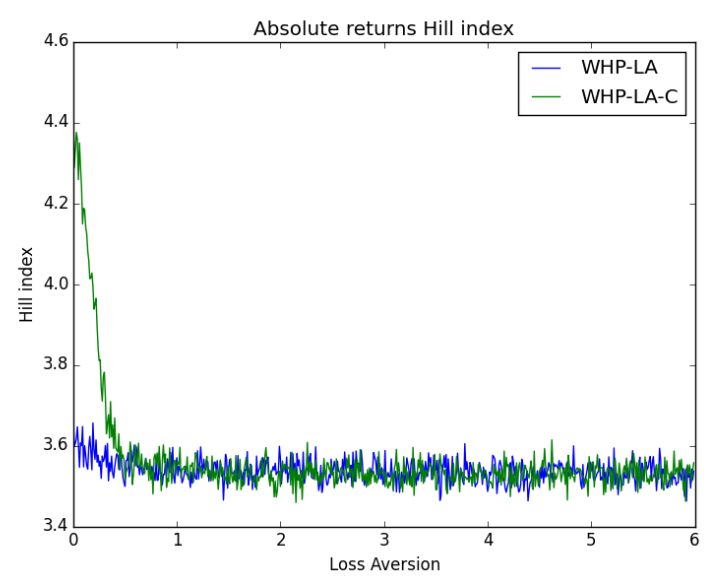

(d) Hill tail index

Figure 3: Impact of loss aversion parameter on agents' interactions and returns.

periods (days), the market is occupied by fundamentalists $9.53 \%$ of the time. In contrast, for the WHP or WHP-LA models, the chartists almost never fully disappear from the market $\left(n_{f} \rightarrow 1\right.$ in less than $1.2 \%$ of the simulations). Since the only difference between the three settings is the loss aversion for chartists, we conclude that it is this behavioural bias that pushes these agents entirely out of the market. This is an interesting result since even zero intelligence strategies, where agents buy or sell randomly, have been shown to survive the competition in agent-based settings (Ladley, 2012). Therefore, in the complex setting of financial markets, where strategies compete with each other, a behavioural bias can be powerful enough to push the agents out of the market.

Note that so far we have kept the loss aversion parameter $\lambda$ equal to 2.25 , as it is reported by prospect theory literature. With this level of aversion we have seen that if just one type of agents are biased (WHPLA-C model), they tend to disappear from the market. However, when we make all agents loss averse (WHP-LA model), the effects induced by this heuristic are not strong enough to produce major disruptions in the model. That is because of agents' demands making their wealth change continuously from positive to negative levels. With almost half of time making or losing money, the effects of loss aversion are not prominent.

Given this, it is interesting to determine what levels of loss aversion are required to produce any effects 
on agents' interactions. To this end, we build on our previous work (Pruna et al., 2016) and vary the magnitude of the loss aversion parameter in both WHP-LA and WHP-LA-C models to test its impact not only on agents' market fractions, but also on the system's macro dynamics. Accordingly, we increase the loss aversion parameter from Equation (8), considering equally spaced values in the interval $\lambda \in[0,6]$. In Figure 3 (a) we plot the loss aversion parameter $(\lambda)$ against the median value of the fundamentalists' market fraction $\left(n_{f}\right)$ for both WHP-LA and WHP-LA-C. Observe how rapidly the fundamentalists' market fraction increases with the increase of our loss aversion parameter. Specifically, if $\lambda>2$ the chartists usually occupy less than $0.5 \%$ of the market.

To analyse the actual impact of the loss aversion parameter on the agents' interaction in greater detail, we look at the end intervals of the market fractions. To this end, Figure 3 (b) plots the median percentage of the simulations that see the chartists completely disappearing from the market $\left(n_{f} \rightarrow 1\right)$. It is clear that more and more chartists are being thrown out of the market as their loss aversion parameter increases. In the case of WHP-LA-C model, where just the chartists are biased, the $5 \%$ threshold is hit at $\lambda=2.43$. Hence, the interesting dynamics appear for a loss aversion parameter close to the value of 2.25 reported by Tversky and Kahneman (1992). At this level, the fundamentalist occupy the market $11.94 \%$ of the time, or approximately 3.25 years of the 27 years simulation time.

On the other hand, when we make all agents biased in the WHP-LA model, we observe how chartists disappear from the market at larger values of loss aversion. In this case, the $5 \%$ level is hit at $\lambda=3.10$. It is important to note that even when all agents are considered to be biased (not reported here in detail), the chartists don't survive the competition. Moreover, the chartists disappearance is not a rare event, the fundamentalist occupying the market $12 \%$ of the simulation time of 6966 periods. Interestingly, the fundamentalists always stay in the market, dominating it. Even when we consider a model with biased fundamentalists and unbiased chartists, the fundamentalists market fractions still dominate the market and chartists survive, similar to what we see in the WHP model.

We now turn our attention to the implications of loss aversion on the simulated returns series produced by our behavioural models. In Figure 3 (c) we plot the kurtosis of returns for different levels of loss aversion. We observe how for our WHP-LA and WHP-LA-C models, the kurtosis rapidly increases as the loss aversion parameter becomes larger. Moreover, in both cases the kurtosis stabilises at a value around 2.2 for all $\lambda>1$. However, while for WHP-LA we see an increase of kurtosis of 10\%, for the WHP-LA-C model the kurtosis has a $50 \%$ increase from 1.40 to 2.20 .

Furthermore, in Figure 3 (d) we consider the Hill tail index of absolute returns as a function of the loss aversion parameter. This provides a quantitative measure of the heaviness of the tail for the distribution of returns. The Hill index stabilises around a value of 3.60 for a loss aversion parameter $\lambda>1$, experiencing a considerable $18 \%$ decrease for the WHP-LA-C model.

As we have demonstrated, loss aversion can have a considerable impact on our behavioural agent-based model. On one hand, if only the chartists are loss averse, even the standard value of $\lambda=2.25$ is powerful enough to drive them out of the market. On the other hand, if both fundamentalists and chartists are biased, the same effect is observed when we employ a sufficiently larger loss aversion parameter $(\lambda>3.10)$. Overall, the number of chartists disappearing from the market grows as we increase the magnitude of loss aversion. These results are in line with behavioural finance literature arguing that investors' systematic biases can lead to unexpected market behaviour, with deviations from efficiency and rationality. Finally, we have shown that loss aversion has an impact on the distribution of returns, increasing their peakiness and the heaviness of the tails.

\section{Stylized Facts}

In this section we explore the statistical properties generated by our WHP-LA model, keeping the loss aversion parameter fixed at the usual value $\lambda=2.25$. We show that our behavioural model matches a rich set of empirically observed stylized facts, demonstrating one of the main objectives of ABM. An in-depth analysis of the time series generated by the model will be performed. First, we demonstrate the existence of the most discussed properties, namely the absence of autocorrelations, heavy tails, volatility clustering 


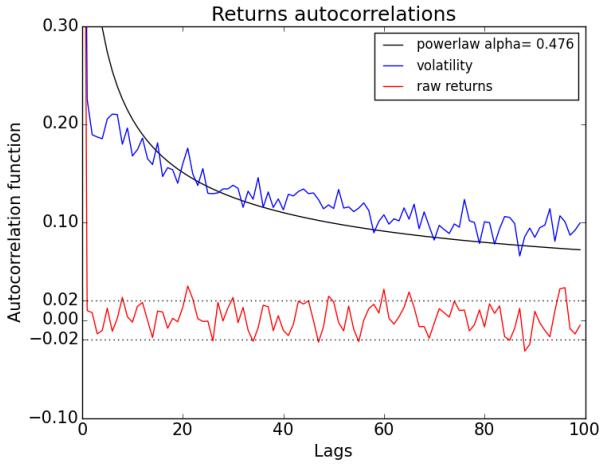

(a) Autocorrelation function

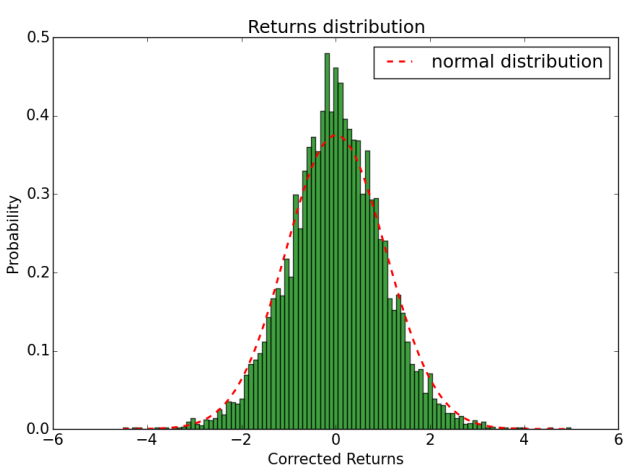

(c) Volatility corrected returns distribution

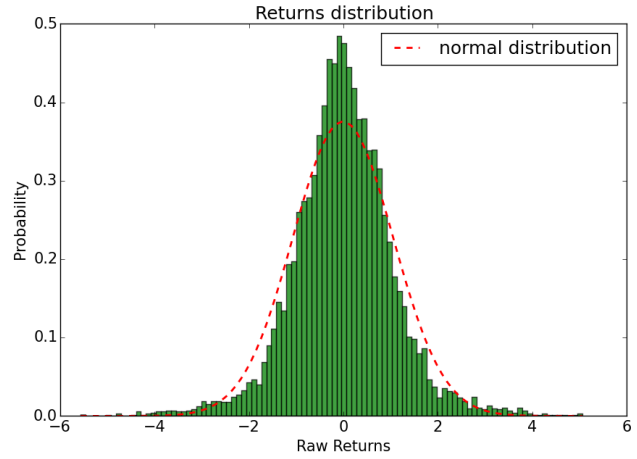

(b) Returns distribution

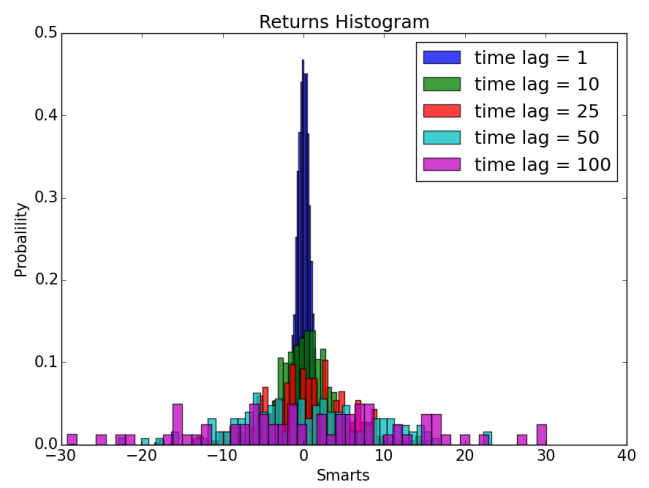

(d) Aggregate Gaussianity

Figure 4: Returns' stylized facts.

and long memory. Moreover, our model is able to match some of the less discussed stylized facts such as conditional heavy tails, aggregate Gaussianity, gain-loss asymmetry, volume power-law, long memory and volume-volatility relations.

\subsection{Absence of autocorrelations, volatility clustering, long memory and heavy tails}

The lack of predictability of price series is one of the most distinctive characteristics of financial time series, closely related to the property stating that price movements do not exhibit any significant autocorrelation (Cont, 2001). The absence of autocorrelations can be easily observed by plotting the ACF of raw returns (see Figure 4a), demonstrating that price changes are not autocorrelated.

However, the absence of autocorrelations does not rule out the possibility of non-linear dependencies of returns. When analysing financial time series, volatility measured as non-linear representations of returns exhibit a much higher positive autocorrelation that persists over time. This is a quantitative signature of volatility clustering: large price variations are more likely to be followed by large price variations, as observed in Figure 1 (d). In the ACF of absolute returns plotted in Figure 4 (b), we observe a positive autocorrelation that persists over time, indicating the presence of volatility clustering.

In addition, we can test the presence of volatility clustering by investigating how well a standard GARCH(1,1) model fits the returns. In Figure 5 (a) and (b) we plot the residuals time series of our simulated and empirical returns, their autocorrelations and partial autocorrelations and the QQ and Probability plots comparing the distribution of returns with a normal distribution. Both residuals are similar to white noise, having no significant autocorrelation. However, there is obvious conditional heteroskedasticity (conditional 


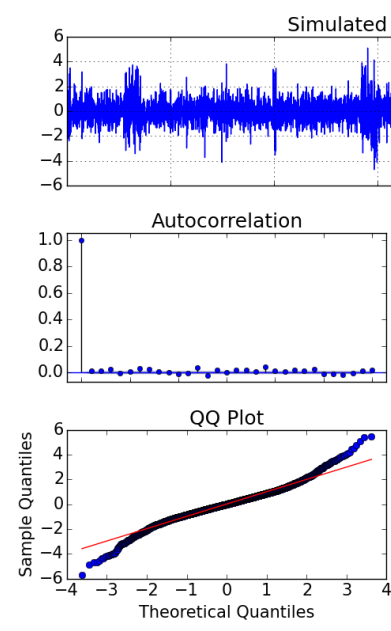

(a) Simulated returns
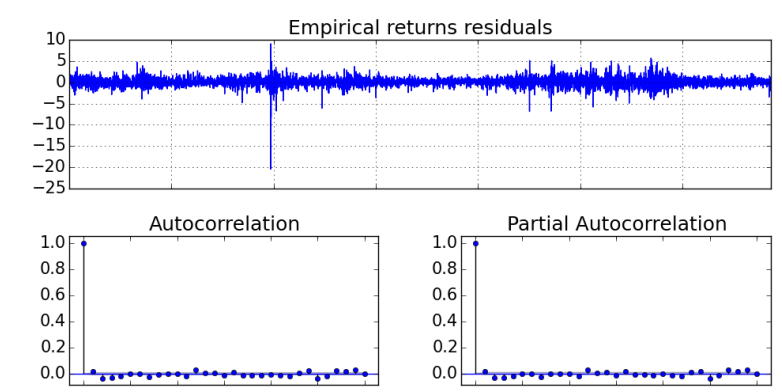

Probability Plot
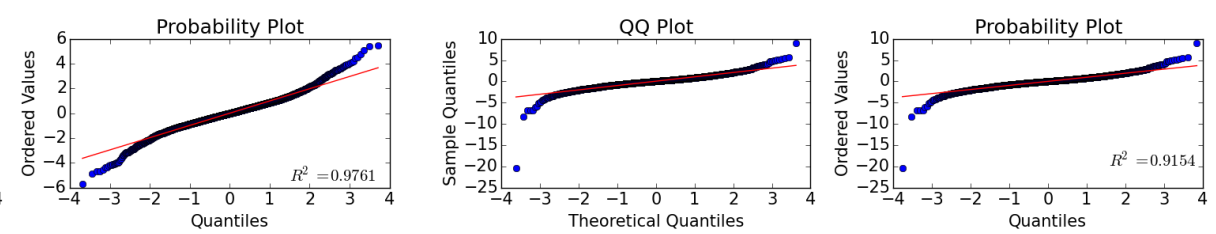

(b) Empirical returns

Figure 5: Residuals time series analysis.

Table 4: Excess kurtosis and skewness as we increase the time lags at which returns are computed.

\begin{tabular}{|l|l|l|l|l|l|}
\hline time lag & 1 & 10 & 25 & 50 & 100 \\
\hline kurtosis & 2.32 & 1.94 & 1.42 & 0.95 & 0.56 \\
\hline skewness & -0.0046 & 0.0026 & 0.0349 & 0.038 & 0.0763 \\
\hline
\end{tabular}

volatility) that the model has not captured. The shapes of the QQ and Probability plots indicate that the processes are close to normality but with heavy tails.

A further property closely related to volatility clustering is the long memory effect, specifically addressing the decay in the ACF of absolute returns. We usually say that a process exhibits long memory if it has a slow decay, similar to a hyperbolic function. One way of observing the decay in the ACF of volatility is by fitting a power law of the form $A / r^{\alpha}$. We notice that a fit can be obtained, with an exponent $\alpha=0.476$ (see Figure 4 (a)), in line with empirical studies (Cont, 2001).

Next, we study the distribution of returns (see Figure 4 (b)) and observe its deviations from the normal distribution. Our simulated returns, similarly to the ones of real financial assets, are bell shaped but contain more mass in the peak and tails than normal. Specifically, an excess kurtosis of 2.35 implies a peakiness bigger than normal and a slow asymptotic decay of the probability density function. This non-normal decay is the so-called heavy tail (LeBaron and Samanta, 2005).

\subsection{Conditional heavy tails and aggregate Gaussianity}

In this section, we consider further properties observed in financial time series that are rarely matched by agent-based models. We begin with the so-called conditional heavy tails of returns. It states that even after correcting returns for volatility clustering (e.g. via GARCH-type models), the residuals time series still has heavy tails (Cont, 2001). However, the tails are less heavy than the unconditional distribution. A visual representation of the property can be observed in Figure 4 (c), where we plot the distribution of the residual returns after correcting for volatility clustering via a $\operatorname{GARCH}(1,1)$ model. Compared with the normal distribution superimposed on it, we see that the residuals of returns have a higher peak and longer tails, confirmed by an excess kurtosis of 0.60 . At the same time, the tail is less heavy than the one of raw returns.

Clearly, the distribution of returns has excessive kurtosis and heavy tails, with higher peak than the normal distribution and power law tails. However, this behaviour changes as we increase the time scale over which returns are calculated. Specifically, the distribution of returns changes its shape, becoming 


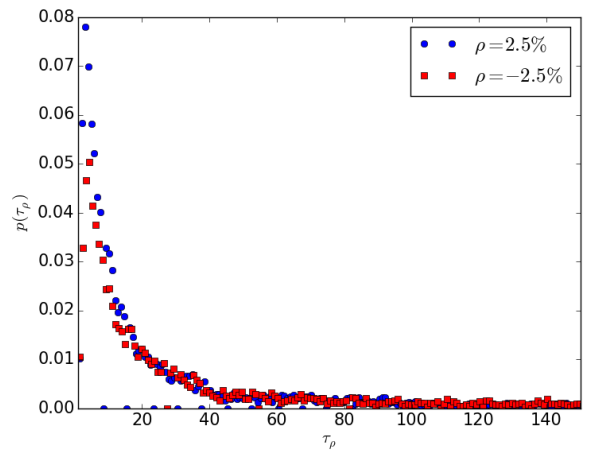

(a) Investment horizon distribtion for a return level $|\rho|=2.5 \%$. The insert is the optimal investment horizon $\tau_{\rho}^{*}$ for positive and negative levels of returns

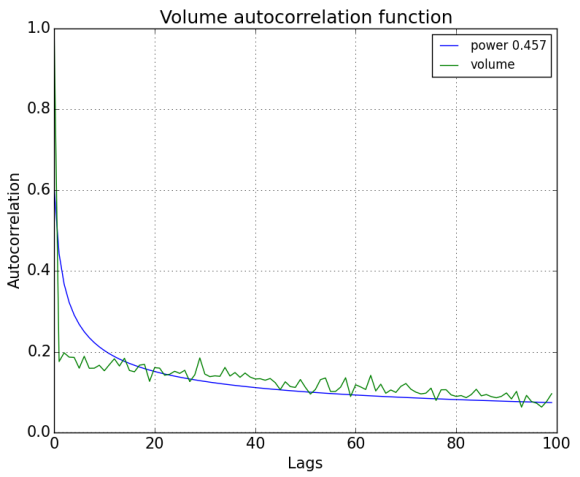

(c) Volume autocorrelation function

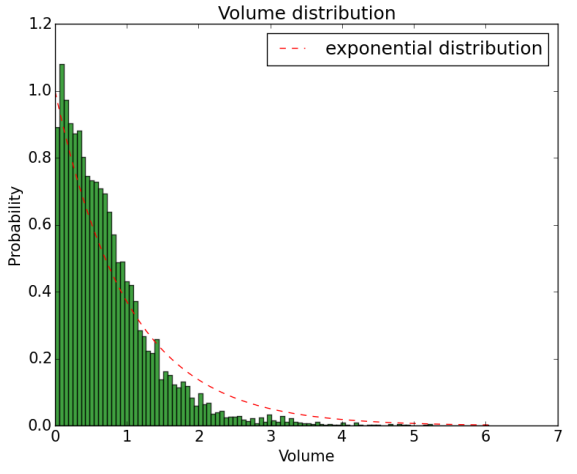

(b) Volume distribution

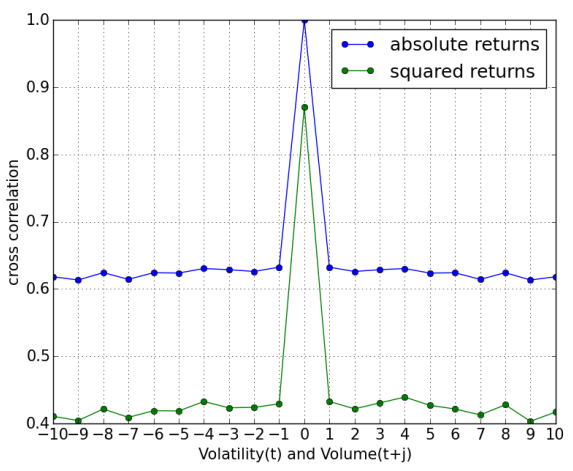

(d) Volume-volatility relations. Cross correlation between volatility measured as absolute retuns (blue) and squared returns (green) and volume distribution.

Figure 6: Gain-loss asymmetry and volume related stylized facts.

more like the normal distribution. This stylized fact, known as the aggregate Gaussianity (Cont, 2001), can be observed in Figure 4 (d), where we plot the distribution of returns calculated for different time lags $(\tau=1,10,25,50,100)$. Note how the distribution of returns becomes more like the normal one as we increase the time lags, with their excess kurtosis decreasing (see Table 4).

\subsection{Gain-loss asymmetry}

In order to obtain a deeper understanding of the fluctuations of prices and returns, Simonsen et al. (2002) proposed a different kind of approach involving inverse statistics. In the analysis of financial data, the inverse question can be formulated as follows: For a given return on an investment, what is the typical time span needed to obtain this return. Specifically, the gain/loss asymmetry of returns states that while the maximum of inverse statistics for a positive level of returns occurs at a specific time, the maximum for the same negative level of returns appears earlier (Jensen et al., 2003) . For the first time in agent-based modelling, we will match this stylized fact with a 2-type design model.

In more detail, let $\pm \rho$ be the level of return, where the positive or negative sign corresponds to a gain or a loss, respectively. It is kept fixed and one looks for the shortest waiting time after $t, \tau_{ \pm \rho}(t)$, for which the returns is above or below the predefined $\pm \rho$. The distribution of waiting times for gains $\tau_{+\rho}$, and loses 
$\tau_{-\rho}$, is denoted $p\left(\tau_{ \pm \rho}\right)$ and corresponds to a fixed return level $\rho$. Such a distribution typically goes through a maximum $\tau_{\rho}^{*}$, called the optimal investment horizon (Simonsen et al., 2002).

In Figure 6 (a) we plot the probability distribution function, $p\left(\tau_{ \pm \rho}\right)$, of waiting times $\tau_{ \pm \rho}$, with a return level $\rho=2.5 \%$. We can observe the asymmetry between simulated investment horizon distributions. In particular, for a negative level of return, there is a higher probability to short investment horizons, as compared to what is observed for the positive one. On average, while the optimal investment time for a negative return of $\rho=-2.5 \%$ is 20 days, for the same level of positive returns we obtain an optimal investment time of 23 days.

\subsection{Volume power-law, long memory and volume-volatility relations}

We now turn our attention to volume, yet another highly discussed financial topic. Some of the empirical quantitative properties related to volume include a power-law behaviour, long memory and correlation to volatility. We will demonstrate all these stylized facts with our behavioural model. We define the volume at time $t$ as the total absolute demand at $t$ of both fundamentalists and chartists. In Figure 6 (b) we can see that the volume distribution has a bigger decay than the exponential distribution superimposed on it, indicating a power-law behaviour. Moreover, an excess kurtosis of 6.036 reinforces this behaviour.

In Figure 6 (c) we plot the autocorrelation function of volume. We observe a power-law decay with an exponent 0.457 . The slow decay is a clear presence of long-memory of volume. Another widely used method for testing the long memory effect is by using the Hurst exponent. We obtain the value of 0.68 for the Hurst exponent of the distribution of simulated volume, in line with most empirical studies reporting a vale in the interval $[0.5-1]$ for long memory processes. Finally, the dependence between volatility and volume traded has been noticed and documented across different financial instruments at different time scales. Specifically, the relationship between the two market descriptors can be observed via the cross correlation function. In Figure $6(\mathrm{~d})$ we observe a significantly positive cross correlation between volatility and volumes. This means that a small (large) trading volume is accompanied by a small (large) change in volatility. It is important to note that all measures of volatility are positively correlated with volume and that the dependence remains significant as we increase the time lag.

\section{Conclusions}

Heterogeneous agent-based models that rely on simple trading strategies have proven themselves very efficient in generating important dynamics of real financial markets. Indeed, the fundamentalists vs. chartists models have been shown to successfully capture empirically observed traders' behaviour. Moreover, a well-defined agent-based model able to match the financial markets' common properties is an important tool for testing various behavioural and economical theories and understanding how they influence the interactions between agents and prices.

In this paper, we described, evaluated and extended one of the most successful approaches in capturing real-life market dynamics. We presented a new behavioural model of asset pricing where the agents are loss averse. In the new setting, we observed major differences in the movements of the agents' market fractions compared to unbiased scenarios. Specifically, for certain levels of loss aversion, the biased chartists tend to be driven out of the market at different points in time. Additionally, by comparing the statistics of the base model without loss aversion and our new behavioural model, we have demonstrated how certain levels of this bias change the distribution of returns, leading to a more pronounced volatility clustering and a distribution of returns with higher peaks and heavier tails. Therefore, we can link our findings with the behavioural finance literature, which states that investors' systematic biases lead to surprising market behaviour, instabilities and errors.

Furthermore, the main objective of agent-based financial modelling is to propose an alternative to the apparent randomness of financial markets, trying to explain the most important properties of financial data. Specifically, we are interested in simple structures that can reproduce the empirical findings to a high degree and which are quantitatively close to the real ones. Trying to measure how close a model is to its empirical counterparts, we make use of two different statistics. First, the distance between their summary statistics 
(moments) is measured by a quadratic loss function. For the second, we used the concept of (joint) moment coverage ratio. Here, with the help of Monte Carlo runs, we count the number of times where all the simulated statistics are contained in the empirical confidence intervals. This can be seen as a quantitative measure of how may often the data from a model and the real market cannot be told apart. With a p-value of $31.06 \%$ and a coverage ratio of $55.37 \%$, the introduction of loss aversion improves the degree to which the model explains the real data, compared to the same model without loss aversion.

We offer both a quantitative and a qualitative analysis of the simulated asset price series, its returns, volume traded and volatility. We provide a wide range of tests and arguments and demonstrate the presence of a rich set of stylized facts including absence of autocorrelations, heavy tails, conditional heavy tails, volatility clustering, long memory of returns, aggregate Gaussianity, gain-loss asymmetry, volume power law, long memory and volume-volatility relations. By doing so, our behavioural model is the first one to match such a rich set of the stylized facts.

For future work, we would like to apply loss aversion in a setting that models individual agents instead of considering the state-of-the-art representative agent scenario. Furthermore, we will investigate the relationship between the stationarity of returns and the method of estimating the model's parameters. Moreover, we are interested in investigating how other behavioural factors such as the disposition effect, gossip or waves of optimism and pessimism influence the way agents interact with each other. To this end, we make use of well-defined agent-based models to test the impact of various behavioural biases on the market. Unfortunately, the high complexity of the real financial markets makes it impossible to test such implications directly on them. By employing agent-based models, we offer an alternative to the apparent randomness of the markets, detecting the consequences of different biases and heuristics, which otherwise cannot be identified.

\section{Acknowledgements}

This work was supported by the EPSRC ORCHID project (EP/1011587/1). The authors acknowledge the use of the IRIDIS High Performance Computing Facility in the completion of this work.

\section{References}

Alfarano, S., Lux, T., Wagner, F., 2005. Estimation of agent-based models: the case of an asymmetric herding model. Computational Economics 26, 19-49.

Alfi, V., Cristelli, M., Pietronero, L., Zaccaria, A., 2009. Minimal agent based model for financial markets i. The European Physical Journal B 67, 385-397.

Asparouhova, E., Bossaerts, P., Plott, C., 2003. Excess demand and equilibration in multi-security financial markets: The empirical evidence. Journal of Financial Markets 6, 1-21.

Barberis, N., Huang, M., Santos, T., 1999. Prospect theory and asset prices. Technical Report. National bureau of economic research.

Barde, S., et al., 2015. Direct calibration and comparison of agent-based herding models of financial markets. Technical Report. School of Economics, University of Kent.

Beja, A., Goldman, M.B., 1980. On the dynamic behavior of prices in disequilibrium. The Journal of Finance 35, 235-248.

Bikhchandani, S., Sharma, S., 2000. Herd behavior in financial markets. IMF Economic Review 47, $279-310$.

Bouchaud, J.P., Potters, M., 2003. Theory of financial risk and derivative pricing: from statistical physics to risk management. Cambridge university press.

Brennan, T.J., Lo, A.W., 2011. The origin of behavior. The Quarterly Journal of Finance 1, 55-108.

Brock, W.A., Hommes, C.H., 1997. A rational route to randomness. Econometrica: Journal of the Econometric Society , 1059-1095.

Chen, S.H., Chang, C.L., Du, Y.R., 2012. Agent-based economic models and econometrics. The Knowledge Engineering Review $27,187-219$.

Chen, Z., Lux, T., 2018. Estimation of sentiment effects in financial markets: A simulated method of moments approach. Computational Economics 52, 711-744.

Chiarella, C., 1992. The dynamics of speculative behaviour. Annals of operations research 37, $101-123$.

Chiarella, C., Dieci, R., He, X., 2008. Heterogeneity, market mechanisms, and asset price dynamics. Quantitative Finance Research Centre Research Paper. 
Chiarella, C., He, X.Z., 2002. Heterogeneous beliefs, risk and learning in a simple asset pricing model. Computational Economics 19, 95-132.

Chiarella, C., He, X.Z., 2003. Heterogeneous beliefs, risk, and learning in a simple asset-pricing model with a market maker. Macroeconomic Dynamics 7, 503-536.

Chiarella, C., He, X.Z., Hommes, C., 2006. A dynamic analysis of moving average rules. Journal of Economic Dynamics and Control 30, 1729-1753.

Cont, R., 2001. Empirical properties of asset returns: stylized facts and statistical issues .

Cont, R., Kukanov, A., Stoikov, S., 2014. The price impact of order book events. Journal of financial econometrics 12 , 47-88. De Bondt, W., 2005. The psychology of world equity markets. Edward Elgar.

Fama, E.F., 1970. Efficient capital markets: A review of theory and empirical work. The journal of Finance 25, $383-417$.

Farmer, J.D., Joshi, S., 2002. The price dynamics of common trading strategies. Journal of Economic Behavior \& Organization 49, 149-171.

Franke, R., 2009. Applying the method of simulated moments to estimate a small agent-based asset pricing model. Journal of Empirical Finance 16, 804-815.

Franke, R., Westerhoff, F., 2011. Estimation of a structural stochastic volatility model of asset pricing. Computational Economics 38, 53-83.

Franke, R., Westerhoff, F., 2012. Structural stochastic volatility in asset pricing dynamics: Estimation and model contest. Journal of Economic Dynamics and Control 36, 1193-1211.

Franke, R., Westerhoff, F., 2016. Why a simple herding model may generate the stylized facts of daily returns: explanation and estimation. Journal of Economic Interaction and Coordination 11, 1-34.

Frankel, J.A., Froot, K., 1990a. Exchange rate forecasting techniques, survey data, and implications for the foreign exchange market. Technical Report. National Bureau of Economic Research.

Frankel, J.A., Froot, K.A., 1990b. Chartists, fundamentalists, and trading in the foreign exchange market. The American Economic Review , 181-185.

Gehrig, T., Menkhoff, L., 2004. The use of flow analysis in foreign exchange: exploratory evidence. Journal of International Money and Finance 23, 573-594.

Gilli, M., Winker, P., 2003. A global optimization heuristic for estimating agent based models. Computational Statistics \& Data Analysis 42, 299-312.

Hommes, C.H., 2006. Heterogeneous agent models in economics and finance. Handbook of computational economics 2, 11091186.

Jensen, M.H., Johansen, A., Simonsen, I., 2003. Inverse statistics in economics: the gain-loss asymmetry. Physica A: Statistical Mechanics and its Applications 324, 338-343.

Kahneman, D., 2011. Thinking, fast and slow. Macmillan.

Keynes, J.M., 1937. The general theory of employment. The quarterly journal of economics 51, $209-223$.

Ladley, D., 2012. Zero intelligence in economics and finance. The Knowledge Engineering Review 27, $273-286$.

LeBaron, B., 2006. Agent-based computational finance. Handbook of computational economics 2, $1187-1233$.

LeBaron, B., Samanta, R., 2005. Extreme value theory and fat tails in equity markets. Available at SSRN 873656 .

Lo, A.W., 2007. Efficient markets hypothesis .

Lo, A.W., MacKinlay, A.C., 1988. Stock market prices do not follow random walks: Evidence from a simple specification test. Review of financial studies 1, 41-66.

Lux, T., Marchesi, M., 2000. Volatility clustering in financial markets: a microsimulation of interacting agents. International journal of theoretical and applied finance 3, 675-702.

Pruna, R.T., Polukarov, M., Jennings, N.R., 2016. An asset pricing model with loss aversion and its stylized facts. Computational Intelligence for Financial Engineering \& Economics (CIFEr), IEEE Symposium Series , 1-8.

Samuelson, P.A., 1965. Proof that properly anticipated prices fluctuate randomly. IMR; Industrial Management Review (pre-1986) 6, 41.

Shefrin, H., 2002. Behavioral decision making, forecasting, game theory, and role-play. International journal of forecasting 18, $375-382$

Shiller, R.J., 2003. From efficient markets theory to behavioral finance. The Journal of Economic Perspectives 17 , 83-104.

Simon, H.A., 1955. A behavioral model of rational choice. The quarterly journal of economics , 99-118.

Simonsen, I., Jensen, M.H., Johansen, A., 2002. Optimal investment horizons. The European Physical Journal B-Condensed Matter and Complex Systems 27, 583-586.

Taylor, M.P., Allen, H., 1992. The use of technical analysis in the foreign exchange market. Journal of international Money and Finance 11, 304-314.

Thaler, R.H., 2005. Advances in behavioral finance. volume 2. Princeton University Press.

Tversky, A., Kahneman, D., 1974. Judgment under uncertainty: Heuristics and biases. science 185, $1124-1131$.

Tversky, A., Kahneman, D., 1991. Loss aversion in riskless choice: A reference-dependent model. The quarterly journal of economics , 1039-1061.

Tversky, A., Kahneman, D., 1992. Advances in prospect theory: Cumulative representation of uncertainty. Journal of Risk and uncertainty 5, 297-323.

Winker, P., Gilli, M., Jeleskovic, V., 2007. An objective function for simulation based inference on exchange rate data. Journal of Economic Interaction and Coordination 2, 125-145.

Wooldridge, M., Jennings, N.R., 1995. Intelligent agents: Theory and practice. The knowledge engineering review $10,115-152$. 